filtering before we have a fluid in which we can with certainty affirm the presence of peptone. Not very uncommonly it happens that the result of this proceeding in cases of dyspepsia is negative, yet we are not thereby enabled to conclude that pepsin is deficient unless albumoses also are present. It becomes necessary to carry our investigation a stage further by employing our filtered gastric contents as a digestive agent in artificial digestive experiments. This work necessarily takes up a good deal of time, and I do not suggest that every busy practitioner can investigate his cases so completely.

A great number of experiments has demonstrated that atony of the stomach, involving delay in the transfer of the gastric contents into the intestine, is the most constant, the earliest, and the most important factor in the production of the common dyspepsia of women. The defective movements of the muscular wall lead to incomplete admixture of the gastric juices with the ingesta. By and by the stomach becomes slightly dilated; a residue of food remains in the stomach antil the next meal is taken, and this liquid organic matter, which contains normally many micro-organisms and is deficient in its natural antiseptic, hydrochloric acid, undergoes fermentative decompositions, giving rise to the formation of lactic and butyric acids, which cause irritation of the gastric mucous membrane, discomfort and pain, and are attended by the evolution of gases, which cause flatulence and often smell offensively. A stomach in such a condition is very prone to attacks of subacute gastritis. This inflammatory reaction is slight at first, but it weakens the resistance of the membrane, renders it more prone to acute or subacute exacerbations, manifested by what are called bilious attacks, and, in course of time, determines atrophy of the glands of the stomach, with consequent diminution of the gastric juice, and as it extends through the mucous and submucous coats to the muscular layer further weakens this, and leads to increased atony and some degree of permanent dilatation. When this is established the vicious circle is completed and all the evils I have enumerated are intensified and the stomach troubles become very serious. The initial evil-atony of the wall of the stomach-arises from many conditions-anæmia, overwork, want of sleep, and anxiety--which are the lot of so many women in the course of the duty of bringing up a family ; it arises from superlactation, want of sufficient food, confinement to the house, and irregular meals, from acute diseases, and want of proper rest. good food, and fresh air during convalescence. Does not the recital of these causes remind one too forcibly of the common lot of most of the women in our great towns; not only the wives of artisans or of the middle class, of clerks, warehousemen, and shop assistants, but of the wives of many who are in fairly comfortable circumstances, but on whom sickness makes demands which they are not in a position to meet with all the resources of wealth? I do not think we need wonder that these cases are so deplorably common, and that they continue to get worse instead of better under the persistence of unfavourable conditions and the routine prescription of digestive nostrums; and simple atony of the stomach due to debility, originating as has been described, constitutes the essential and sole lesion in the majority of women suffering from so-called dyspepsia.

\section{A CASE OF "SUPPURATIVE PYLEPHLE- BITIS" IN WHICH LAPAROTOMY WAS PERFORMED.}

BY FREDERICK TREVES, F.R.C.S. ENG.,

SURGEON TO, AND LECTURER ON SURGERY AT, TIIE LONDON HOSPITAL EXAMINER IN SURGERY, UNIVERSITY OF CAMBRIDGE.

For the opportunity of publishing this case I am indebted to the kindness of Mr. Frederick Parsons of Wimbledon, under whose care the patient was. The subject of the somewhat remarkable experience now to be recorded was a young lady aged fifteen, who was seized on the evening of Sept. 17th, 1892, with the symptoms of perityphlitis. Her home was in Canada, but she was at the time a pupil at a school in England. She had recently returned from Canada and had landed from the steamer about ten days before the date mentioned. The points worthy of note in her previous history were these. She had always enjoyed robust health, but had been the subject of somewhat troublesome constipation. Four years previously she had had a slight attack of perityphlitis, which was attended with high fever, but which soon yielded to castor oil. Again, two years ago, she had a similar attack after an ocean passage. This was attended by constipation and was almost immediately relieved by aperients. One of her brothers had had an attack of perityphlitis. During her voyage across the Atlantic her appetite had been excellent, and she bad in no way checked it. Not only had she eaten very well at all her meals, but she had consumed a quantity of nuts, including cocoa-nut, and no inconsiderable amoun of "candy" during the leisure intervals of the day. She was menstruating during the voyage and appears to have totally neglected her bowels. During the ten days which elapsed between her arrival in Ingland and the onset of the attack she had been in her usual good health, and, according to her own account, her bowels had acted regularly. The attack came on at night with abdominal pain and vomiting. The pain was about the lower part of the abdomen and was at first inconsiderable; it increased, however, and by noon on the following day (Sept. 18th) was severe and more diffused. At this time she was seen by Mr. Parsons, who found the abdomen to be a little distended and exceedingly tender. The pulse was 90 , small, and regular; the temperature was $100^{\circ} \mathrm{F}$. the tongue was thickly coated. Some Dover's powder and grey powder were given, as well as a little nepenthe. A hot fomentation was applied to the abdomen, and a limited milk diet was ordered. These measures soon gave relief to the pain ; the romiting ceased and did not again appear until some days later. The temperature reached $1026^{\circ}$ on the 19th (the third day) and $104^{\circ}$ on the morning of the fourth day. On the $19 \mathrm{th}$ two enemata were given, which brought away a few scybala. On the 21 st (the fifth day) a more copious motion was passed, and the temperature dropped to normal. On the sixth day the bowels were freely relieved four times, and on the seventh day the patient felt almost well, her temperature on the evening of that day being $98.8^{\circ}$. The only part of the abdomen in which any tenderness remained was the right iliac fossa. On the eighth day, at 5 P M, the patient had a rigor of some sevexity, the temperature rising to $103^{\circ}$. She also vomited once. On the next day the pain and tenderness in the abdomen had returned; there was now considerable meteorism, the patient complained greatly of distension, and she had pain and difficulty in micturition. The bowels were kept well open by means of enemata and an occasional dose of castor oil. On the ninth day there was one rigor at 1.40 P.M. (temperature $1026^{\circ}$ ); on the tenth day there were four rigors (highest temperature $104 \cdot 4^{\circ}$ ), and on the eleventh day two rigors (temperature $1028^{\circ}$ ). On the last-named day Sir William Broadbent saw the patient with Mr. Parsons. He diagnosed the case as one of stercoral ulcer and ascribed the high temperature to the absorption of poisonous products. At that time the bowels were still acting daily; the pain and tenderness were on the whole less and were once more almost limited to the right iliac fossa. There was no vomiting; the distension of the abdomen continued. There was no interference with the breathing, but there was still difficulty in micturition. There was no rigor on the twelfth, fourteenth, and fifteenth days, but there were two on the thirteenth. On this day the patient complained for the first time of pain in the back, just below the last rib of the right side. Under the influence of treatment the bowels were acting daily, and at this date a considerable quantity of granular matter was passed which appeared to be composed of masticated nuts. The patient was taking her food fairly well, but was still restricted to a slop diet. She was very weak and was troubled with profuse perspiration and with occasional shooting pains in the stomach. There had been no vomiting since the eighth day. The abdomen was still distended and tympanitic. From the tenth to the twenty-ninth day there was little new to record. The patient was gradually becoming worse and was losing: strength rapidly. She seemed to be utterly prostrate and miserable. The range of the temperature during these days presented remarkable features. On almost every day there was a severe and exhausting rigor. These occurred at very irregular hours. The six rigors last recorded were noted at the following times-viz., 5 P.M., 7 A.M , 2 P.M., 8 P.M., 4 A.M., and 9 A.M. The rigors were most distressing, causing the child to cry out in terror, and they were followed by profuse perspiration. There was now vomiting of a copious bile-stained fluid, mixed with mucus, which was associated with most of the rigors. The bowels also had become loose, many fluid motions being passed daily, with a 
good deal of mucus, but no blood. As time passed on, the motions came to resemble those met with in typhoid fever. The child was much emaciated, and some delirium was noted for the first time on the twenty-sirth day. The skin became a dull-grey colour. There was never any jaundice. No albumen was discovered in the urine except in an amount which the high fever might account for. There was at no time swelling of the feet, and there was no cdema of the abdominal wall, no ascites, and no hiccough. On the nineteenth day there was some pain in the right chest, which "caught her breath." It soon passed off, and at no time was there any lung complication. The abdomen remained distended and tympanitic. There was still a little tenderness over the cæcum, but the chief pain was over the situation of the liver. The whole of the skin covering the hepatic area appeared to be hypersensitive, and the tenderness was especially marked behind. The liver was enlarged, but the tenderness and the existing meteorism rendered its exact definition difficult. Throughout the child continued to take food, in spite of almost constant nausea and the vomiting which was associated with the rigors. On the thirtieth day the patient was again seen by Sir William Broadbent. It was decided that, inasmuch as the symptoms suggested the existence of an abscess or abscesses in the liver, an exploratory incision should be made into the abdomen. This incision I carried out on the following day, Oct. 17th-i.e., thirty-one days after the occurrence of the first symptoms. The child now looked miserably ill; she was much emaciated and very prostrate, the forehead was wrinkled, and she appeared to be in a state of unceasing anxiety. The pain was complained of as being "all over," but the only place where tenderness was very marked was in the region of the liver. The abdomen was still distended and uniformly tympanitic. Nothing abnormal could be felt in the right iliac region. I made an incision in the right semilunar line, commencing it first below the costal margin. The intestine was moderately distended with gas, the distension being notably in the coils of the lesser bowel. There was no peritonitis and no ascites, and at no part within the abdomen did I encounter any adbesions, with the exception of a few about the upper part of the cæcum. The cæcum itself presented to the touch nothing abnormal; the appendix was normal and freely movable. The gall-bladder exhibited no morbid changes and contained about half an ounce of bile. The liver was remarkable; it was considerably and evenly enlarged. It projected at once into the wound and extended an inch and a half or more below the margins of the costal cartilages. So far as its surface could be examined no adhesions were discovered. It was normal in colour and felt to the touch almost as soft as a lung. This loss of its normal firmness was very striking. All that part of the surface of the liver which was exposed by the operation was dotted over with minute yellow specks. They were perfectly round and were comparable in size to the holes made in paper by the prick of an ordinary pin. They were to be seen, but not to be felt. I roughly marked out a square inch on the upper surface of the liver and counted three of these little specks within the space defined. They were pretty evenly distributed. I examined, so far as was possible, the region of the fissure of the portal vein, but did not note anything peculiar. I omitted to make an examination through the foramen of Winslow. I made no attempt to inspect the main mesenteric vessels; such inspection would have been almost impossible. There was no evidence of tuberculous disease in any part. The abdominal wound was closed in the usual way. The patient's recovery was rapid and uninterrupted. By the evening of the day of the operation she was already much relieved. The pain went, and the tenderness disappeared. She had no more rigors. The nausea and sickness ceased, the meteorism disappeared, and the bowels became regular. The temperature, however, remained high for some time. For the first twelve days after the operation it ranged between $99.2^{\circ}$ and $103^{\circ}$, but the chart ceased to exhibit the abrupt up-and-down movement. For the next twelve days it ranged between $99^{\circ}$ and $102^{\circ}$, the mean being about $100^{\circ}$. After the twenty-sixth day from the date of the operation the temperature remained normal. The child has kept in most excellent health ever since and has had no abdominal symptoms of any kind. At the present timesome eighteen months after her illness-she is in sound
health, has grown considerably, and has become stouter than she was before she was seized with the last attack of typhlitis.

Remarks.-- The case was one evidently of typhlitis or peri- typhlitia followed by pylephlebitis. It may be here stated that the child had never had ague and had never lived in a malarious district. Her parents, who had come over from Canada on hearing of her illness, gave precise information upon this point. Quinine had no effect upon either the rigors or the fever. The case is of interest, in the first place, as affording evidence that all cases of perityphlitis do not depend upon mischief in the appendix, and that even when there are repeated attacks that little process may not be concerned in them. There is little doubt but that the pathogenesis of the present attack was as follows. There had been constipation and the eating of a large quantity of indigestible food. An accumulation formed in the cæcum and, partly by pressure, partly by chemical and bacteriological irritation, induced a (so-called) stercoral ulcer. This ulceration of the bowel would allow the bacterium coli commune to reach the peritoneum and. by exciting some peritonitis, to lead to the phenomena of perityphlitis. It is well known that alceration of the appendix may lead to all the symptoms of perityphlitis without there being any visible perforation or gangrene. Indeed, in the exudation upon the surface of an apparently sound appendix containing a concretion the bacterium coli commune has been found. The term "typhlitis" bas little claim to be allowed to exist, but such a case as the one under discussion affords some apology for its occasional use. It is needless to say that in those examples of perityphlitis which depend distinctly upon the appendix constipation and irregularities in diet are often the immediate causes of an attack, and that the patient appears to be able to ward off a relapse by attention to his bowels and care in the choice of his food. In the present instance, however, the perfectly normal condition of the appendix and of its mesentery must exempt that little process from complicity in the illdoings which surrounded it. That genuine typhlitis or perityphlitis (independently of mischief in the appendix) is very rare has been amply demonstrated, but such a case as the one just related affords evidence that it actually has an existence. It would appear that in this young girl there was more widespread ulceration of the colon in the later period of the attack, as shown by the diarrhoea, the tenderness of the abdomen, and the large quantity of mucus which was passed. The term "pylephlebitis" is applied to phlebitis of the portal vein or its main tributaries, thrombosis of the affected vessels being of necessity associated therewith. Very good systematic accounts of this disease have been given by $D_{r}$. Bartholow in "Pepper's System of Practical Medicine," 1 and by Dr. Chauffard in the "Traité de Médecine," edited by MM. Charcot, Bouchard, and Brissand. ${ }^{2}$ These accounts differ in no material respect. Two forms of pylephlebitis are recognised-the adhesive and the suppurative. In the adhesive form the affected veins show the phenomena of simple thrombosis. The causes given are com. pression of the portal vein by cirrhosis, tumour or gumma of the liver, perihepatitis, sub-hepatic peritonitis, and nlcer of the alimentary canal. The chief symptoms are pain over the liver, ascites of a very high grade, distension of the superficial abdominal veins, enlargement of the spleen, and passive congestion of the gastro-intestinal mucous membrane, leading to vomiting (possibly with hæmatemesis) and to diarrhoa, in which blood may be noticed in the motions; there is usually no jaundice. The symptoms may persist for weeks or even for several months; " but a fatal termination," writes Dr. Bartholow, "is, sooner or later, inevitable in all cases." I hare met with one case, however, of adhesive pylephlebitis which is an exception to this formidable prognosis. The patient was under the care of my friend Mr. Boyter Johnson, and I had the opportunity of seeing him, at frequent intervals, for many months. He was examined also by Dr. Ralfe and Dr. John Anderson. The patient, aged twenty-two, was an officer in the army. In November, 1890, when in India, he had dysentery. This was followed in thirty days by symptoms which were ascribed first to peritonitis and then to abscess of the liver. He had considerable ascites, much pain over the liver, any vomiting, with (later on) occasional hæmatemesis and diarrhoea with bloody stools. The liver was enlarged, and in February, 1891, the spleen was described as being "double its size." There was fever throughout, with occasional rigors. He went to Australia in that month, and in June the greatid distended abdomen was tapped. This was followed by a laparotomy, in the course of which the gall-bladder was unfortunately opened and a biliary fistula was produced. The 
Liver was seen to be normal, Between June and November the abdomen was tapped eight times. The largest amount remored was five quarts. In November the patient came to England. He was then much emaciated, the ascites was considerable, and the distension of the superficial veins of the abdomen reached a degree I have never seen equalled. There was never any jaundice. The biliary fistula was still active. Cnder a treatment which consisted in the main of absolute rest in bed, a suitable diet, and massage the patient made 2. perfect recovery ; the fistula closed, the ascites vanished, and in September, 1892, the patient rejoined his regiment.

The suppurative form of pylephlebitis is said to be most commonly due to ulceration and suppuration of some part of the intestinal tube, and to most commonly lead to multiple abscesses of the liver. Typhlitis and ulcers of the rectum appear to have been the starting points in most of the cases. To follow Dr. Bartholow's account, the inflammatory or ulcerative action extends to and involves the walls of the reins. Coagulation of the blood in the vessel ensues, and the clot nndergoes a series of changes resulting in the formation of emboli, which, carried into the main carrent, are subsequently lodged in the bepatic capillaries. The following are the symptoms described. The onset of the inflammation of the vein is announced by a rigor or chill. When the liver becomes involved there is pain, which is asually complained of in the right hypochondriac and epigastric regions. The pain is accompanied by a well-localised tenderness on pressure. "The fever succeeding the chill is decided and in some cases may attain to extraordinary height. The fever intermits or remits with a more or less profuse perspiration. The febrile phenomena are similar in their objective expression to malarial fever, but there is an important difference in respect to the periods of recurrence of the chills. The paroxysms are very irregular as to time; there may be a daily seizure at different hours, or there may be several chills on the same day ; in other words, the paroxysms have the pymmic characteristics rather than the malarial. After a time the intermittent phenomenon ceases and there occurs a remission merely, the exacerbation being preceded by chilliness and succeeded by sweating. The sweats are characteristically profuse and exhausting...... The maxima may be from $103^{\circ}$ to $105^{\circ}$ or even to $106^{\circ}$. When the pain and chill come on, disturioances of the digestive organs ensue.....The appetite is lost, the stomach becomes irritable, and vomiting is a usual incident. Sometimes the disgust for food is extreme, and the nausea and romiting are almost incessant. The vomited matters consist of a watery mucus mixed with thin bile after a time, and now and then of a bloody mucus. ...... Diarrhcea is a usual symptom, and three-fourths of the cases of pylephlebitis are free from jaundice." Dr. Bartholow concludes by stating that dilatation of the superficial veins of the abdomen is rare and that an enlargement of the liver is to be noted when the formation of abscesses occurs. He adds that the disease ends fatally in from two weeks to three months. Tith the exception of the prognosis, the account given in the swo text-books named might have been drawn from the case I bave now recorded. In no single particular are the clinical phenomena observed and the systematised description at Fariance. I assume that the thrombosis involved some considerable portion of the superior mesenteric vein. It is not necessary to conclude that the clot extended into the trunk of the portal vein; indeed, there is substantial evidence against that possibility. It is clear that fragments of the softened clot were carried into the portal circulation and produced the numerous yellow foci with which the liver was dotted. As to whether the term "abscess" should be applied to these little specks is open to question. It may be surmised that in each minute nodule there would have been found a colony of the bacterium coli commune. In a few cases of operation for relapsing typhlitis I have found outside the cæcum a not inconsiderable quantity of custard-like matter, which was certainly neither pus nor a product of the inspissation of pus. That it was an inflammatory product was evident, and that the colon bacillus had been concerned in its development was probable. I mention this in order to support the impression that the colon bacillus, although pyogenic, is capable of producing an inflammatory exudation which, in its physical characters, falls short of actual pus. That the sittle foci in the liver would have led to abscess in the course of time there is, I imagine, little doubt; and if the nomenclature in vogue be followed I assume that no other term can be applied to the present case than that of "suppurative pylephlebitis." In what way the progress of the case was affected by the laparotomy it is impossible to say. The curative effect of a simple incision into the abdomen, which is often little short of miraculous, has yet to be explained. It has been shown that in the treatment of the ascitic form of tuberculous peritonitis the best result have followed a mere cut into the peritoneal cavity with evacuation of the fluid, but without either irrigation or drainage. Dr. Willian White, in his very admirable monograph on the "Curative Effect of Operations per se," has collected a remarkable series of cases, in which, quite unexpectedly, a vast improvement in the patient's state or a complete cure of the disorder has attended a simple exploratory incision. In many instances large solid tumours have disappeared or have undergone an astounding diminution. I have recently had under my care in the London Hospital a young man who had a large solid tumour in the left side of the abdomen, which extended from the groin to the costal cartilages and from the loin to beyond the median line. An exploratory incision proved it to be sarcomatous. The abdomen was closed, and the tamour forthwith began to shrink until it resolved itself into two nodules, one the size of an adult's fist and the other that of a large hen's egg. The wasting continued, and fresh growths appeared outside the abdomen, but the improvement within the cavity was maintained.

In the Lettsomian lectares on Peritonitis I have ventured to saggest that these and other not less remarkable results of simple incision may depend upon an alteration brought about in the state of pressure within the abdomen, whereby the circulation is amended and the conditions associated with absorption are substantially modified. Precise evidence in support of this suggestion is, I must confess, still wanting.

Wimpole-street, $\mathbf{W}$

\section{THE QUANTITATIVE DETERMINATION OF COLOUR VISION.}

BY ROBERT BRUDENELL CARTER, F.R.C.S. ExG., CONSULTING OPITHALMTC SURGEON TO ST. GEORGE'S HOSPITAL.

IT is well known that the condition commonly called colour blindness, whether congenital, and coextensive with the field of vision, or acquired and limited to a portion of the field, may be either complete or incomplete; that is to say, that the subject of the defect may be totally insensitive to all colours or to some colours, or that he may be sensitive to them in a degree which does not attain the normal acuteness of perception. I have long thought it desirable that the degrees of acuteness of colour pision should be measured and expressed numerically, just as is done with the degrees of acuteness of form vision; and the object of the present paper is to bring before the profession an instrument which I have contrived for this purpose, and by which, as it appears to me, satisfactory measurements may be obtained. In examining; say 500 men, in order to ascertain whether they are fit for employment on railways or in navigation, the examiner will find a great majority with colour sense of normal acuteness, and a minority, usually equal to about 2 or 3 per cent. of the whole, whom it will at once be his duty to reject. $\mathrm{He}$ will find another minority concerning whom he may entertain reasonable doubt. Their colour sense is defective, but is it so defective as to disqualify them for the situations which they seek? That this question is a real and practical one may be shown by the example of a young gentleman lately under my observation who was partially blind for green. He was liable to error when dealing with diluted colours, but not when dealing with full colours. He was rejected on the score of defective colour vision for one branch of military service, and was immediately afterwards accepted for another branch in which colour vision was certainly quite as much required as in the former. My object is to render some standard attainable, so that authorities, either of the public services or of railways, may be able to say "We require colour vision of such or such a degree of acuteness," and so that examiners may be able to determine whether this standard of acuteness is reached or exceeded. Again, in acquired colour blindness, which, as in the ordinary forms of toxic amblyopia, is generally localised in the centre of the field, it is important to be able to estimate the degree from time to time, so as to obtain evidence of improvement or 